\title{
Reconstruction of the Dead (Grey) Dune evolution along the Curonian Spit, Southeastern Baltic
}

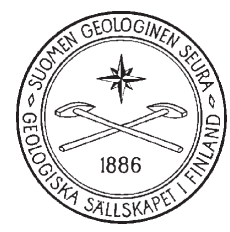

\author{
Nikita Dobrotin ${ }^{1)^{*}}$, Albertas Bitinas ${ }^{2)^{*}}$, Dainius \\ Michelevičıus ${ }^{3)}$, Aldona Damušrte ${ }^{4)}$ and Jonas Mažeika ${ }^{5)}$ \\ 1) Klaipeda University, Coastal Research and Planning Institute, 84 H. Manto St., \\ LT-92294 Klaipeda, Lithuania \\ 2) Klaipeda University, Coastal Research and Planning Institute, Department of \\ Geophysical Sciences, 84 H. Manto St., LT-92294 Klaipeda, Lithuania \\ 3) Vilnius University, Faculty of Natural Sciences, 21/27 Ciurlionio St., \\ LT-03100 Vilnius, Lithuania \\ 4) Lithuanian Geological Survey, 35 S. Konarskio St., LT-03123 Vilnius, Lithuania \\ 5) Nature Research Centre, Institute of Geology and Geography, 13 T. Ševčenkos St., \\ LT-03223 Vilnius, Lithuania
}

\section{Abstract}

One of the unique places in Europe in both environmental and cultural terms is the Curonian Spit - a massive sandy barrier separating the Curonian Lagoon from the Baltic Sea. Straddling both the Lithuanian and the Russian parts, the spit is included into the UNESCO list of cultural heritage monuments. From the geological point of view, it is still an "alive" environment dominated by aeolian deposits. With the help of modern geophysical and geochronological techniques (ground-penetrating radar [GPR] surveys, LIDAR data, and radiocarbon $\left[{ }^{14} \mathrm{C}\right]$ dating), detailed investigations of paleosols were carried out in the Dead (Grey) Dunes massif located between Juodkrante and Pervalka settlements on the Lithuanian half of the Curonian Spit. Several soil-forming generations (phases) during 5800-4500, 3900-3100, 2600-2400, and from 1900 calendar years BP until the present have been distinguished. GPR surveys enabled a series of paleogeographic reconstructions of the massif for different time intervals of its evolutionary history.

Keywords: ground-penetrating radar, paleosols, artifacts, absolute age, C-14, paleogeography, Holocene, Curonian Spit, Lithuania

* Corresponding authors email: nikas.dobrotinas @gmail.com, albertas.bitinas@corpi.ku.lt 


\section{Introduction}

Curonian Spit is a sandy barrier separating the Curonian Lagoon from the Baltic Sea (Fig. 1A \& B). It is one of the unique places in Europe in cultural and geological terms. This landform, both the Lithuanian and the Russian parts, is included into the UNESCO list of cultural heritage monuments. Its dunes are the highest dunes in Northern Europe and reach more than $30 \mathrm{~m}$ in many areas (Gudelis, 1998a). The highest "Senosios smukles" (i.e. "Old Inn") dune, also known as Vicekrugo dune, is $67.2 \mathrm{~m}$ height and is situated a few kilometres to the south from Preila (Fig. 1B). All the biggest dune's massifs are forming so-called Great Dune Ridge that stretches along the entire lagoon coast of the spit (Fig. 1C). The history of geological development of the Great Dune Ridge is poorly known except the fact that during the XVIXIX centuries, due to strong impact of human activity (clearcutting of forests in the greater part of the Curonian Spit), an aeolian activity was extremely high. As a result, 14 villages have been buried beneath the sandy dunes along the Curonian Lagoon coast of the spit (Gudelis, 1998a; Bučas, 2001). After that, starting in the beginning of XIX century, a substantial part of the Curonian dunes was artificially forested. It arrested some aeolian activity and protected the fishing villages against sand invasion. At the same time, starting in 1805 , the artificial foredune was formed along the entire length of the marine coast of the Curonian Spit. This foredune protected (as a barrier) the inner part of the spit from the seashore sand drift. However, parts of aeolian massifs are not yet covered by vegetation and represent ideal natural "laboratories" where investigations of aeolian processes are still possible. Due to recent aeolian processes, a number of buried paleosols, which are significant indicators of stability phases during the dune development, are outcropping in many unvegetated areas. This fact, as well as a new possibility of effective investigations of sandy dunes by ground-penetrating radar (GPR) (Buynevich et al., 2007a, 2007b, 2009), stimulated an idea to reconstruct the geological evolution of the Great Dune Ridge. One of still active aeolian massifs, the so-called Dead (Grey) Dunes, which are located between Juodkrante and Pervalka settlements and have a status of Nature Reserve (Fig. 1. B, C), have been chosen as the focus area for our studies. The investigations were carried out in 20112012 along a $15 \mathrm{~km}^{2}$ region.

\section{Investigation of paleosols}

The Curonian Spit dunes are not of the same age they were formed during different periods of time. This is indicated by the layers of paleosols inside the dune massifs. J. Schumann (1861) was the first who mentioned this fact and later Hess von Wichdorff (1919) described in detailed the old dune distribution, formation, and aspects of their evolution. The monograph also contained a detailed description of ancient forest soils (Wichdorff, 1919). The detailed data on paleosols was presented in $\mathrm{K}$. H. Paul's (1944) monograph where he published a scheme of outcropping paleosols located to the south from Nida settlement.

More detailed scientific investigations of the Curonian Spit dunes started only after World War II and were generally focused on geomorphologic studies and recent dynamics of dune development. Significant input into the studies of dunes and paleosols was made by a Lithuanian scientist V. Gudelis (1989-1990, 1998a, 1998b, etc.), who was an initiator of the thorough investigations of the entire spit. He carried out visual mapping of paleosols in some areas (Gudelis et al., 1993) and proposed three so-called "aeolodynamic stages" of the Curonian Spit dune development: 1) intensive dune formation period (5000-4000 years BP); 2) dune stabilization period (from 4000 to 500 years $\mathrm{BP}$ ), and 3) intensive regeneration period (after 500 years BP) (Gudelis, 1998a).

The results of pollen analysis revealed that formation of paleosols had started at the end of the Atlantic Period (i.e. about 5000 calendar years BP) and continued during the Sub-Atlantic and SubBoreal (Gudelis et al., 1993; Gaigalas et al., 1991, Moe et al., 2005). The conclusions of pollen analysis were confirmed by the results of radiocarbon $\left({ }^{14} \mathrm{C}\right)$ dating of paleosols (Chichiagova \& Cherkinski, 


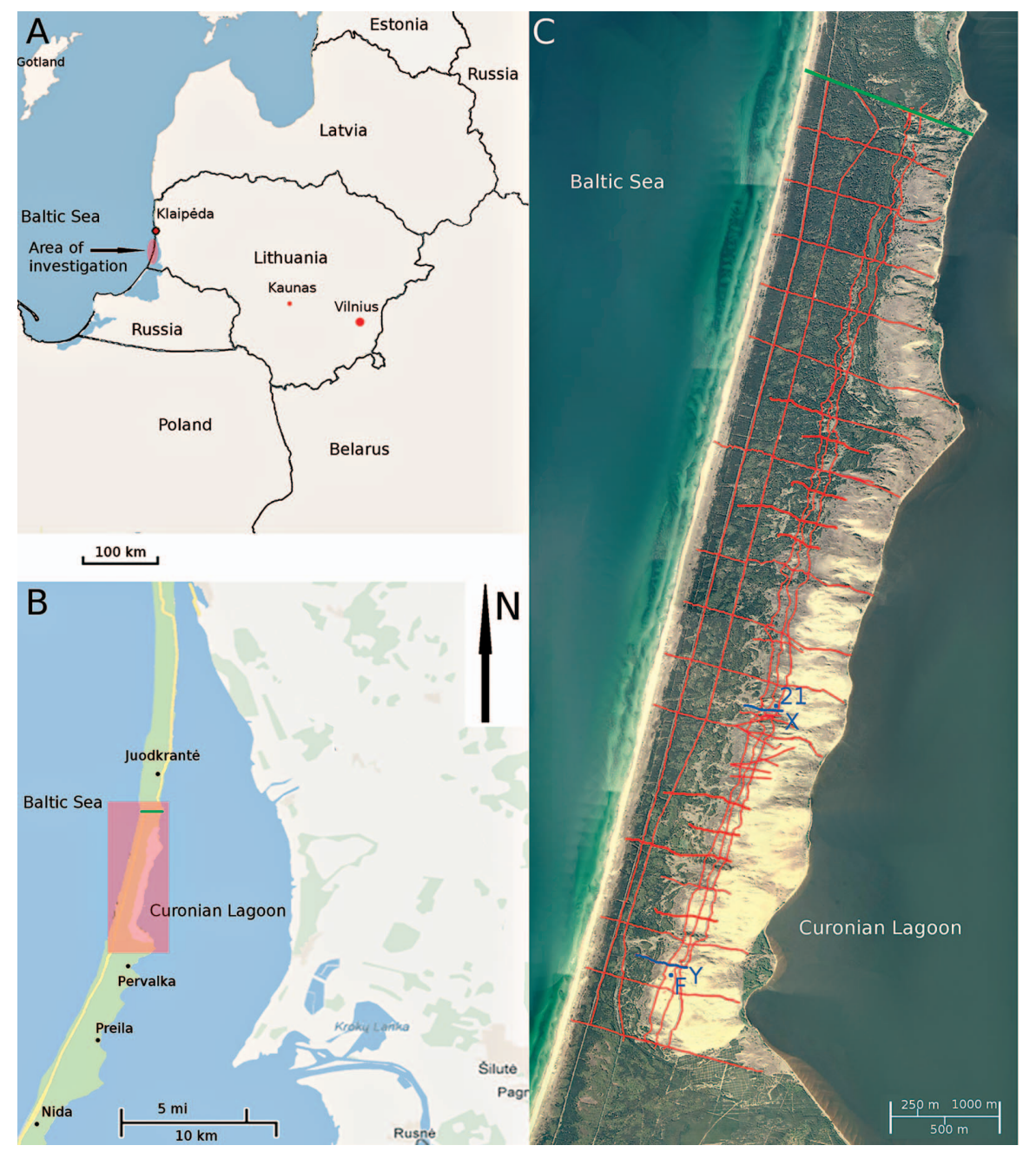

Fig. 1. Location of study area. Rectangle in B corresponds to enlarged area in section $\mathrm{C}$. The location of groundpenetrating radar (GPR) profile across the Curonian Spit (Fig. 2) is marked by green line (B, C). Red lines (C) are showing the GPR profiles grid. Blue lines and points show location of the GPR profiles (marked as $\mathrm{X}$ and $\mathrm{Y}$ ) and exposed paleosols (marked as F and 21) which are shown in Fig. 4.
1988; Gerasimov \& Zavelski, 1980; Gaigalas et al., 1991). In the past decade, GPR imaging (using 250 and $400 \mathrm{MHz}$ antennas) was carried out at several sites along the Dead (Gray) Dunes aeolian massif (Buynevich et al., 2007a, 2007b). Recently, based on the chronological dates of dunes and paleosols, six phases (generations) of paleosol formation have been distinguished by Gaigalas \& A. Pazdur (2008): 4.6-4.0 kyr BP, 3.4-2.9 kyr BP, 1.9 kyr BP, 1.2$1.0 \mathrm{kyr}$ BP, 0.6-0.4 kyr BP, and 0.29-0.12 kyr BP. The paleosols of different age contain substantial amounts of charcoal. Thus, according to some investigators, forest fires were the main reason for destruction of vegetation, which created favourable conditions for aeolian activity (Gudelis, 1998b; Gaigalas \& Pazdur, 2008).

\section{Methods}

\subsection{GPR surveys}

At the beginning of investigation, the field area (15 $\mathrm{km}^{2}$ ) was surveyed with ground-penetrating radar profiles using a grid with $500 \mathrm{~m}$ profile spacing. Later, part of the study area (in sectors where paleosols were found) was investigated using a denser (200 m) grid (Fig. 1C). Approximately 180 linear $\mathrm{km}$ of subsurface profiles were collected during the study period. GPR surveys were carried out using the RADAR Systems GPR Zond 12-e with 300 $\mathrm{MHz}$ antenna, $400 \mathrm{~V}$ pulse generator (it was selected for the best ratio of resolution and depth) and a time window (range) set to $500 \mathrm{~ns}$. The 
electromagnetic wave refractive index in the sand of the Curonian Spit was obtained by coring data - several boreholes were drilled for this purpose in various places of the spit. The electromagnetic wave average refractive index was adopted as $\mathrm{n} \approx 5.33$ for the dune sands. This allowed vertical resolution of $-22 \mathrm{~cm}$ in unsaturated dune sands. All post-processing was performed with Halliburton Geographix program. Groundwater table and paleosols (Fig. 2) were interpreted based on their strong signal returns and characteristic geometry in radargrams. In some cases, buried paleosols have the same shape as sand layers enriched by heavy minerals (Buynevich et al., 2007a). Thus, a number of established reflectors on the radargrams were identified using control excavations and drillings. Real topography of GPR profiles, as well as altitudes of groundwater table and paleosols, were determined using LIDAR images (Fig. 3). During this procedure, the groundwater table was flattened and paleosol surfaces began to depict the real morphology of paleodunes.

\subsection{Radiocarbon $\left({ }^{14} \mathrm{C}\right)$ dating}

During this study, thirteen bulk samples for radiocarbon $\left({ }^{14} \mathrm{C}\right)$ analysis were collected from the different paleosols (Table 1). Pre-treatment of the samples included crushing and Acid-Alkali-Acid (AAA) washing to remove carbonate and humic acid contamination. The remaining bulk organic carbon was used for benzene production (Kovaliukh \& Skripkin, 1994). The specific ${ }^{14} \mathrm{C}$ activity in benzene was measured using the liquid scintillation counting (LSC) method described by Gupta \& Polach (1985), Arslanov (1985), and using a Tri-CarbOे 3170TR/SL in the Radioisotope Research Laboratory of the Institute of Geology and Geography of

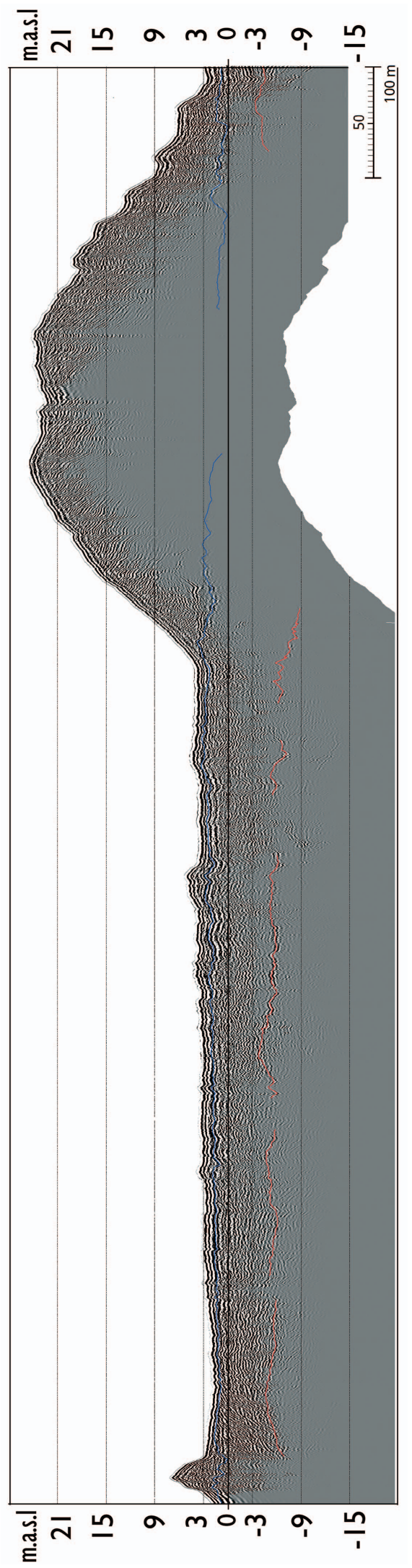

Fig. 2. Ground penetrating radar (GPR) profile across the Curonian Spit (for location see Fig. 1, B, C). The groundwater table is indicated by blue line; red line shows the probable contact between the medium-grained marine sand (above) and the finegrained, organic matter enriched lagoon sand (below). 

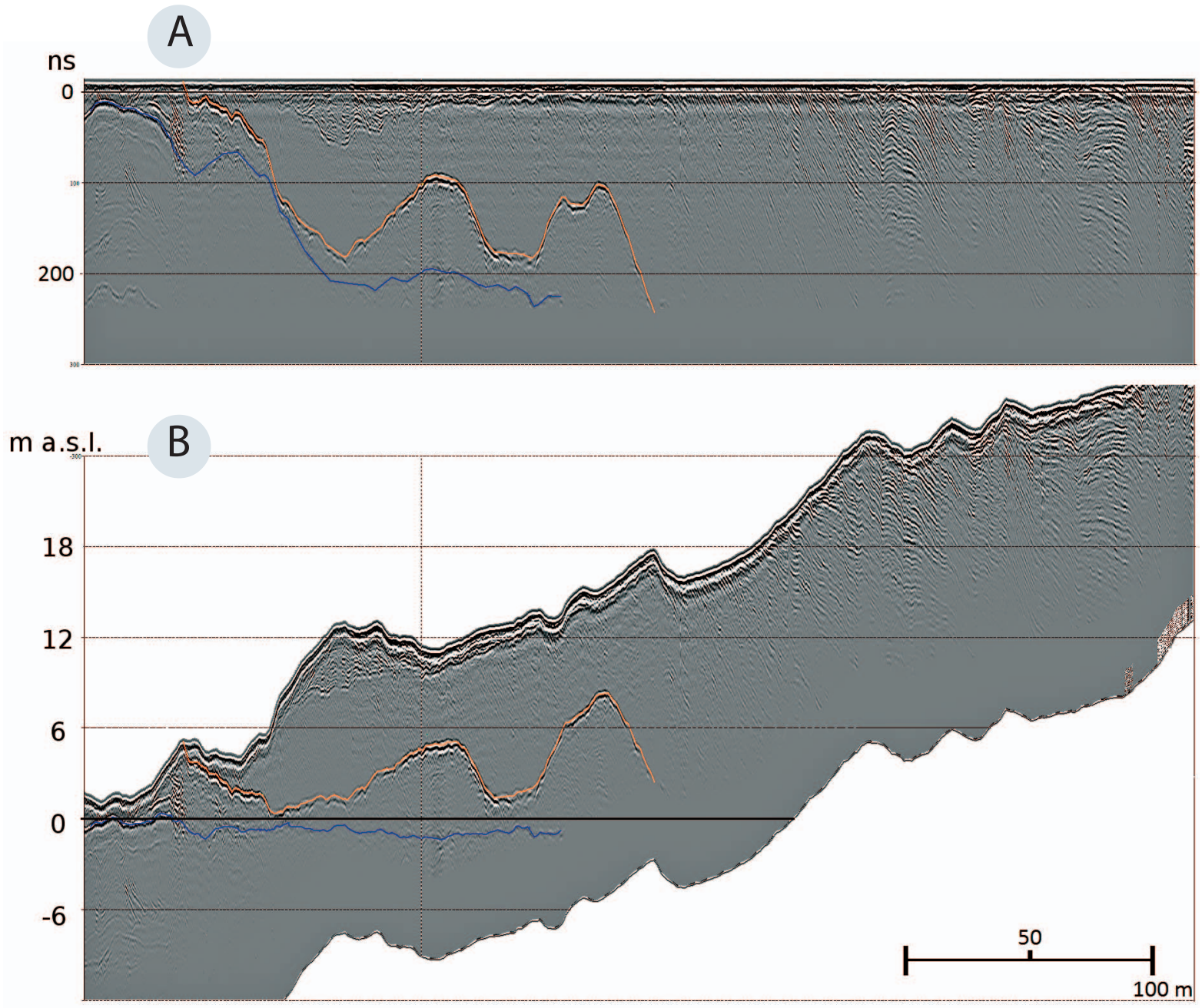

Fig. 3. Primary view of ground penetrating radar (GPR) profile $(\mathrm{A})$ in nanoseconds ( $100 \mathrm{~ns} \approx 6.5$ meters; wave refractive index $n \approx 5.33$ ), and the same profile with altitudes corrected according to LIDAR data (B). The groundwater table is indicated by the blue line. The buried paleosol is shown in orange.

the Nature Research Centre in Vilnius, Lithuania. For a complete picture of paleosol chronology, a number of previous radiocarbon data were compiled from the literature. Several still unpublished AMS and bulk samples data were also used (Table 1, Bitinas et al., unpublished; Buynevich et al., unpublished; the mentioned data are under preparation for publication in the frame of the other scientific projects linked with the Curonian Spit geology). A few published radiocarbon dates that were received during archaeological investigations on the Curonian Spit were also used. The majority of dating results, especially those analyzed several decades ago, were published as uncalibrated data (conventional radiocarbon age). Thus, due to unification of the radiocarbon chronology, all radiocarbon dates were recalibrated using the ${ }^{14} \mathrm{C}$ calibration program OxCal v. 3.1 (Bronk Ramsey et al., 2001) and the calibration curve IntCal09 (Reimer et al., 2009).

\subsection{Paleogeographic reconstructions}

After the GPR radargrams were corrected according to LIDAR database and the soil-forming generations were distinguished, all paleosols were correlated 
Table 1. Results of radiocarbon $\left({ }^{14} \mathrm{C}\right)$ dating of paleosols and artefacts in the Curonian Spit.

\begin{tabular}{|c|c|c|c|c|c|c|c|}
\hline Nr. & Lab. index & $\begin{array}{l}\text { Coordinates } \\
\text { WGS84 / } \\
\text { location }\end{array}$ & $\begin{array}{l}\text { Samplingsite, } \\
\text { paleosol code, } \\
\text { depth, etc. }\end{array}$ & $\begin{array}{l}\text { Method, } \\
\text { analysed } \\
\text { matter }\end{array}$ & $\begin{array}{l}\text { Project / } \\
\text { publication }\end{array}$ & $\begin{array}{l}{ }^{14} \mathrm{C} \text { age, } \\
\text { years (BP) } \\
( \pm 1 \sigma)\end{array}$ & $\begin{array}{l}\text { Calibrated age } \\
\text { (1s range) }\end{array}$ \\
\hline \multicolumn{8}{|c|}{ Paleosols } \\
\hline 1. & Vs-2088 & Vingiakope & $\begin{array}{l}\text { "Oldest" } \\
\text { paleosol }\end{array}$ & Bulk, soil humus & This study & $3180 \pm 120$ & $\begin{array}{l}3560-3316 \text { BP }(59.2 \%) \\
3310-3262 \text { BP }(9.0 \%)\end{array}$ \\
\hline 2. & Vs-2214 & $\begin{array}{l}55^{\circ} 26^{\prime} 42^{\prime \prime} \\
21^{\circ} 04^{\prime} 53^{\prime \prime}\end{array}$ & Paleosol-1 & Bulk, soil humus & $-{ }^{-}$ & $3545 \pm 70$ & $3913-3720$ BP (68.2\%) \\
\hline 3. & Vs-2216 & $\begin{array}{l}55^{\circ} 26^{\prime} 55^{\prime \prime} \\
21^{\circ} 04^{\prime} 55^{\prime \prime}\end{array}$ & Paleosol-2 & Bulk, charcoal & $-{ }^{-\prime}$ & $675 \pm 70$ & $\begin{array}{l}606-556 \text { BP }(36.9 \%) \\
668-624 \text { BP }(31.3 \%)\end{array}$ \\
\hline 4. & Vs-2239 & $\begin{array}{l}55^{\circ} 26^{\prime} 28^{\prime \prime} \\
21^{\circ} 04^{\prime} 56^{\prime \prime}\end{array}$ & Paleosol, G & Bulk, wood & $-{ }^{-\prime}$ & $420 \pm 40$ & $\begin{array}{l}518-459 \text { BP }(63.1 \%) \\
348-340 \text { BP }(5.1 \%)\end{array}$ \\
\hline 5. & Vs-2240 & $\begin{array}{l}55^{\circ} 26^{\prime} 35^{\prime \prime} \\
21^{\circ} 04^{\prime} 48^{\prime \prime}\end{array}$ & Paleosol, K & Bulk, charcoal & $-{ }^{-\prime}$ & $1055 \pm 195$ & $1174-788$ BP $(68.2 \%)$ \\
\hline 6. & Vs-2241 & $\begin{array}{l}55^{\circ} 26^{\prime} 29^{\prime \prime} \\
21^{\circ} 04^{\prime} 45^{\prime \prime}\end{array}$ & Paleosol, F & Bulk, charcoal & $-{ }^{-}$ & $3455 \pm 60$ & $\begin{array}{l}3778-3678 \text { BР }(40.5 \%) \\
3828-3788 \text { BР }(16.5 \%) \\
3669-3640 \text { BР }(11.3 \%)\end{array}$ \\
\hline 7. & Vs-2242 & $\begin{array}{l}55^{\circ} 27^{\prime} 40^{\prime \prime} \\
21^{\circ} 05^{\prime} 31^{\prime \prime}\end{array}$ & $\begin{array}{l}\text { Exposure No.13, } \\
\text { depth } 0.6 \mathrm{~m}\end{array}$ & Bulk, wood & $-{ }^{-\prime}$ & $605 \pm 50$ & $\begin{array}{l}648-584 \text { BP }(54.2 \%) \\
568-550 \text { BP }(14.0 \%)\end{array}$ \\
\hline 8. & Vs-2243 & $\begin{array}{l}55^{\circ} 26^{\prime} 31^{\prime \prime} \\
21^{\circ} 04^{\prime} 49^{\prime \prime}\end{array}$ & Paleosol, I & Bulk, charcoal & $-{ }^{-}$ & $1225 \pm 60$ & $\begin{array}{l}1184-1070 \text { BP }(52.0 \%) \\
1240-1204 \text { BP }(16.2 \%)\end{array}$ \\
\hline 9. & Vs-2244 & $\begin{array}{l}55^{\circ} 26^{\prime} 24^{\prime \prime} \\
21^{\circ} 04^{\prime} 42^{\prime \prime}\end{array}$ & Paleosol, D & Bulk, charcoal & $-{ }^{-\prime}$ & $765 \pm 110$ & $\begin{array}{l}796-640 \text { BP }(60.4 \%) \\
590-564 \text { BP }(7.0 \%) \\
891-887 \text { BP }(0.9 \%)\end{array}$ \\
\hline 10. & Vs-2245 & $\begin{array}{l}55^{\circ} 26^{\prime} 09^{\prime \prime} \\
21^{\circ} 04^{\prime} 42^{\prime \prime}\end{array}$ & $\begin{array}{l}\text { Borehole No. 2, } \\
\text { depth } 1.7 \mathrm{~m}\end{array}$ & Bulk, soil humus & $-{ }^{-}$ & $1735 \pm 150$ & $\begin{array}{l}1826-1516 \text { BP }(63.3 \%) \\
1458-1442 \text { BP }(2.3 \%) \\
1860-1850 \text { BP }(1.4 \%) \\
1432-1422 \text { BP }(1.2 \%)\end{array}$ \\
\hline 11. & Vs-2249 & $\begin{array}{l}55^{\circ} 26^{\prime} 53^{\prime \prime} \\
21^{\circ} 05^{\prime} 02^{\prime \prime}\end{array}$ & Paleosol, N & Bulk, soil humus & $-{ }^{-}$ & $1785 \pm 80$ & $\begin{array}{l}1818-1684 \text { BP }(46.6 \%) \\
1678-1615 \text { BP }(21.6 \%)\end{array}$ \\
\hline 12. & Vs-2250 & $\begin{array}{l}55^{\circ} 27^{\prime} 49^{\prime \prime} \\
21^{\circ} 05^{\prime} 32^{\prime \prime}\end{array}$ & $\begin{array}{l}\text { Exposure No.21, } \\
\text { depth } 0.6 \mathrm{~m}\end{array}$ & Bulk, soil humus & $-{ }^{-}$ & $410 \pm 95$ & $\begin{array}{l}523-426 \text { BP }(41.4 \%) \\
392-319 \text { BP }(26.8 \%)\end{array}$ \\
\hline 13. & Vs-2251 & $\begin{array}{l}55^{\circ} 27^{\prime} 41^{\prime \prime} \\
21^{\circ} 05^{\prime} 22^{\prime \prime}\end{array}$ & Paleosol, V & Bulk, soil humus & $-{ }^{-}$ & $930 \pm 90$ & $927-765$ BP (68.2\%) \\
\hline 14. & Vs-1764 & Lesnoje & $\begin{array}{l}\text { Paleosol } \\
\text { TN } 21 / 1\end{array}$ & Bulk, soil humus & $\begin{array}{l}\text { Bitinas et al., } \\
\text { unpublished }\end{array}$ & $2430 \pm 50$ & $\begin{array}{l}2494-2357 \text { BP }(50.5 \%) \\
2682-2640 \text { BP }(14.1 \%) \\
2610-2599 \text { BP }(3.6 \%)\end{array}$ \\
\hline 15. & Vs-1763 & $-{ }^{-}$ & $\begin{array}{l}\text { Paleosol } \\
\text { TN } 21 / 2\end{array}$ & Bulk, soil humus & $-{ }^{-}$ & $1510 \pm 90$ & $\begin{array}{l}1422-1318 \text { BP }(43.6 \%) \\
1515-1459 \text { BP }(21.1 \%) \\
1442-1432 \text { BP }(3.5 \%)\end{array}$ \\
\hline 16. & Vs-1768 & $-{ }^{-}$ & $\begin{array}{l}\text { Paleosol } \\
\text { TN 21/3 }\end{array}$ & Bulk, soil humus & $-{ }^{-}$ & $1780 \pm 80$ & $1815-1614$ BP (68.2\%) \\
\hline 17. & - & $\begin{array}{l}\text { Dead (Grey) } \\
\text { dunes }\end{array}$ & $\begin{array}{l}\text { Paleosol, } \\
\text { NP-V4 }\end{array}$ & AMS, charcoal & $\begin{array}{l}\text { Buynevich et al., } \\
\text { unpublished }\end{array}$ & $3400 \pm 35$ & $\begin{array}{l}3692-3612 \text { ВР }(62.3 \%) \\
3600-3590 \text { ВР }(5.9 \%)\end{array}$ \\
\hline 18. & - & -"- & $\begin{array}{l}\text { Paleosol, } \\
\text { KNP-V4A }\end{array}$ & AMS, charcoal & $-{ }^{-}$ & $2430 \pm 25$ & $\begin{array}{l}2486-2362 \text { BP }(66.9 \%) \\
2648-2645 \text { BP }(1.3 \%)\end{array}$ \\
\hline 19. & - & $-{ }^{-}$ & Paleosol 1 & AMS, charcoal & $-{ }^{-}$ & $780 \pm 30$ & 725-682 ВР (68.2\%) \\
\hline 20. & - & $-{ }^{-}$ & Paleosol 3 & AMS, charcoal & $-{ }^{-}$ & $3130 \pm 40$ & $\begin{array}{l}3399-3327 \text { BP }(60.3 \%) \\
3286-3270 \text { BP }(7.9 \%)\end{array}$ \\
\hline 21. & - & $-{ }^{-}$ & Paleosol 4 & AMS, soil humus & $-{ }^{-}$ & $4990 \pm 40$ & $\begin{array}{l}5748-5655 \text { ВР }(62.4 \%) \\
5844-5830 \text { ВР }(5.8 \%)\end{array}$ \\
\hline 22. & - & -"- & Paleosol & AMS, charcoal & $-{ }^{-}$ & $660 \pm 30$ & $\begin{array}{l}587-565 \text { BP }(35.1 \%) \\
665-644 \text { BP }(33.1 \%)\end{array}$ \\
\hline 23. & - & - & -"- & AMS, charcoal & $-{ }^{-}$ & $1330 \pm 35$ & $\begin{array}{l}1298-1258 \text { BP }(55.0 \%) \\
1202-1186 \text { BP }(13.2 \%)\end{array}$ \\
\hline 24. & 12919 & Vinkis dune & $\begin{array}{l}\text { Vinkis 2, } \\
\text { depth } 0.75 \mathrm{~m}\end{array}$ & Bulk, charcoal & $\begin{array}{l}\text { Gaigalas \& } \\
\text { Pazdur, } 2008\end{array}$ & $420 \pm 45$ & $\begin{array}{l}520-455 \text { BP }(59.9 \%) \\
348-334 \text { BP }(8.3 \%)\end{array}$ \\
\hline 25. & 12920 & $-{ }^{-}$ & $\begin{array}{l}\text { Vinkis } 3, \\
\text { depth } 0.75 \mathrm{~m}\end{array}$ & Bulk, soil humus & $-n^{-}$ & $970 \pm 50$ & $\begin{array}{l}870-797 \text { BP }(45.0 \%) \\
932-898 \text { BP }(23.2 \%)\end{array}$ \\
\hline 26. & 17430 & $-{ }^{-}$ & $\begin{array}{l}\text { Vinkis } 4, \\
\text { depth } \sim 3 \mathrm{~m}\end{array}$ & Bulk, wood & $-{ }^{-}$ & $125 \pm 95$ & $\begin{array}{l}150-56 \text { BP }(30.5 \%) \\
270-186 \text { BP }(26.8 \%) \\
46-12 \text { BP }(11.0 \%)\end{array}$ \\
\hline
\end{tabular}




\begin{tabular}{|c|c|c|c|c|c|c|c|}
\hline 27. & GdS-570 & Nagliai dune & $\begin{array}{l}\text { Naglis 1, } \\
\text { depth } 0.3 \mathrm{~m}\end{array}$ & Bulk, soil humus & $-{ }^{-}$ & $1050 \pm 30$ & $974-930$ BP (68.2\%) \\
\hline 28. & GdS-571 & $-{ }^{-}$ & $\begin{array}{l}\text { Naglis 2, } \\
\text { depth } 0.43 \mathrm{~m}\end{array}$ & Bulk, charcoal & $-\ldots$ & $590 \pm 30$ & $\begin{array}{l}637-592 \text { BP }(51.5 \%) \\
562-547 \text { BP }(16.7 \%)\end{array}$ \\
\hline 29. & GdS-572 & $-{ }^{-}$ & $\begin{array}{l}\text { Naglis } 3, \\
\text { depth } 0.49 \mathrm{~m}\end{array}$ & Bulk, wood & $-\ldots$ & $630 \pm 30$ & $\begin{array}{l}599-560 \text { BP }(42.1 \%) \\
655-631 \text { BP }(26.1 \%)\end{array}$ \\
\hline 30. & Unknown & $\begin{array}{l}\text { Dead (Grey) } \\
\text { dunes }\end{array}$ & Paleosol P1 & AMS, charcoal & $\begin{array}{l}\text { Buynevich } \\
\text { et al., } 2007\end{array}$ & $850 \pm 35$ & $792-725$ BP (68.2\%) \\
\hline 31. & $-{ }^{-}$ & $-{ }^{-}$ & Paleosol P2 & AMS, charcoal & -"- & $1350 \pm 45$ & $\begin{array}{l}1310-1256 \text { BP }(57.0 \%) \\
1202-1185 \text { BP }(9.9 \%) \\
1247-1244 \text { BP }(1.3 \%)\end{array}$ \\
\hline 32. & TUa-4763 & $\begin{array}{l}55^{\circ} 28^{\prime} 25^{\prime \prime} \\
21^{\circ} 05^{\prime} 37^{\prime \prime}\end{array}$ & $\begin{array}{l}\text { Exposure, } \\
\text { depth } 4-10 \mathrm{~cm}\end{array}$ & AMS, charcoal & $\begin{array}{l}\text { Moe et al., } \\
2005\end{array}$ & $735 \pm 40$ & 698-658 BP (68.2\%) \\
\hline 33. & TUa-4762 & $-{ }^{-}$ & $\begin{array}{l}\text { Exposure, } \\
\text { depth } 47-59 \mathrm{~cm}\end{array}$ & AMS, charcoal & $-{ }^{-}$ & $1900 \pm 40$ & $\begin{array}{l}1898-1813 \text { ВР }(67.0 \%) \\
1750-1746 \text { ВР }(1.2 \%)\end{array}$ \\
\hline 34. & Unknown & Pervalka & Paleosol 3 & Bulk, soil humus & $\begin{array}{l}\text { Gaigalas } \\
\text { et al., } 1991\end{array}$ & $560 \pm 70$ & $\begin{array}{l}641-590 \text { BP }(37.5 \%) \\
564-523 \text { BP }(30.7 \%)\end{array}$ \\
\hline 35. & $-{ }^{-}$ & $-\ldots$ & Paleosol 4 & Bulk, soil humus & $-{ }^{-}$ & $1040 \pm 90$ & $\begin{array}{l}1060-902 \text { BP }(56.2 \%) \\
864-828 \text { BP }(9.1 \%) \\
812-800 \text { BP }(2.9 \%)\end{array}$ \\
\hline 36. & $-n^{-}$ & Nida & Paleosol & Bulk, wood & $-{ }^{-}$ & $3470 \pm 70$ & $\begin{array}{l}3834-3680 \text { BР }(60.0 \%) \\
3666-3642 \text { BP }(8.2 \%)\end{array}$ \\
\hline 37. & $n^{-}$ & Pilkopa & $-n^{-}$ & Bulk, wood & - & $290 \pm 90$ & $\begin{array}{l}476-284 \text { BP }(64.6 \%) \\
167-154 \text { BP }(3.6 \%)\end{array}$ \\
\hline 38. & $-{ }^{-}$ & $-{ }^{-}$ & $-\ldots$ & Bulk, wood & $-{ }^{-}$ & $340 \pm 90$ & $480-310$ BP $(68.2 \%)$ \\
\hline 39. & $-{ }^{-}$ & Vingiakope & Paleosol 3 & Bulk, peat & $-\ldots$ & $1200 \pm 90$ & $\begin{array}{l}1186-1054 \text { BP }(47.5 \%) \\
1256-1202 \text { BP }(15.9 \%) \\
1027-1010 \text { BP }(4.8 \%)\end{array}$ \\
\hline 40. & $-{ }^{-}$ & $\begin{array}{l}\text { SW from } \\
\text { Juodkrante }\end{array}$ & Paleosol & Bulk, wood & $\begin{array}{l}\text { Gerasimov } \\
\text { et al., 1980; } \\
\text { Chichagova \& } \\
\text { Cherkasin, } 1988\end{array}$ & $500 \pm 70$ & $\begin{array}{l}632-598 \text { BP }(16.4 \%) \\
560-494 \text { BP }(51.8 \%)\end{array}$ \\
\hline 41. & $-{ }^{-}$ & Vingiakope & Paleosol I & Bulk, wood & $-\ldots$ & $630 \pm 90$ & $664-550$ BP (68.2\%) \\
\hline 42. & $-n^{-}$ & $-{ }^{-}$ & Paleosol II & Bulk, wood & $-\ldots$ & $1140 \pm 90$ & $\begin{array}{l}1145-965 \text { BP (64.5\%) } \\
1170-1158 \text { BP }(3.7 \%)\end{array}$ \\
\hline 43. & $-\ldots$ & $-{ }^{-}$ & Paleosol III & Bulk, wood & $-{ }^{-}$ & $2960 \pm 90$ & $3260-2996$ BP (68.2\%) \\
\hline 44. & $-{ }^{-}$ & $-{ }^{-}$ & Paleosol IV & Bulk, wood & $-\ldots$ & $4025 \pm 90$ & $\begin{array}{l}4645-4410 \text { BP }(58.4 \%) \\
4801-4762 \text { BP }(7.2 \%) \\
4693-4675 \text { BP }(2.7 \%)\end{array}$ \\
\hline 45. & $-{ }^{-}$ & $\begin{array}{l}N \text { from } \\
\text { Pervalka }\end{array}$ & Upper paleosol & Bulk, soil humus & $\begin{array}{l}\text { Gudelis \& } \\
\text { Michaliukaite, } \\
1976\end{array}$ & $2450 \pm 120$ & $\begin{array}{l}2544-2360 \text { BР }(43.2 \%) \\
2700-2635 \text { BР }(15.6 \%) \\
2616-2584 \text { BР }(7.4 \%) \\
2572-2562 \text { ВР }(2.0 \%)\end{array}$ \\
\hline 46. & $-{ }^{-}$ & $-{ }^{-}$ & Lower paleosol & Bulk, soil humus & $-{ }^{-}$ & $4390 \pm 110$ & $\begin{array}{l}5065-4850 \text { BР }(51.2 \%) \\
5271-5184 \text { BР }(15.5 \%) \\
5120-5112 \text { BP }(1.5 \%)\end{array}$ \\
\hline \multicolumn{8}{|c|}{ Archaeological findings } \\
\hline 47. & Vs-321 & Nida & Fireplace & Bulk, charcoal & $\begin{array}{l}\text { Rimantiene, } \\
1999\end{array}$ & $4630 \pm 120$ & $\begin{array}{l}5483-5275 \text { BР }(45.1 \%) \\
5580-5528 \text { BР }(8.4 \%) \\
5180-5123 \text { BР }(8.2 \%) \\
5110-5068 \text { ВР }(6.5 \%)\end{array}$ \\
\hline 48. & Vs-631 & $-{ }^{-}$ & $-{ }^{-}$ & Bulk, charcoal & $-{ }^{-}$ & $4620 \pm 110$ & $\begin{array}{l}5476-5273 \text { ВР }(45.7 \%) \\
5182-5122 \text { ВР }(9.6 \%) \\
5576-5540 \text { ВР }(5.7 \%) \\
5110-5066 \text { ВР }(7.3 \%)\end{array}$ \\
\hline 49. & Hela-2467 & 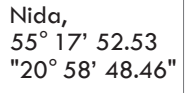 & $\begin{array}{l}\text { Ceramic } \\
\text { shards }\end{array}$ & $\begin{array}{l}\text { AMS, } \\
\text { charred crust }\end{array}$ & $\begin{array}{l}\text { Piliciauskas } \\
\text { et al., } 2011\end{array}$ & $5041 \pm 34$ & $\begin{array}{l}5890-5806 \text { ВР }(50.7 \%) \\
5766-5734 \text { ВР }(17.5 \%)\end{array}$ \\
\hline 50. & Hela-2474 & $-{ }^{-}$ & $-\ldots$ & $-\mu^{-}$ & $-{ }^{\prime \prime}$ & $5005 \pm 34$ & $\begin{array}{l}5750-5661 \text { ВР }(54.1 \%) \\
5855-5829 \text { ВР }(14.1 \%)\end{array}$ \\
\hline 51. & Hela-2469 & ${ }^{-}$ & ${ }^{-}$ & $-\mu^{-}$ & $-\ldots$ & $4946 \pm 34$ & $\begin{array}{l}5714-5642 \text { BP }(55.8 \%) \\
5630-5613 \text { BР }(12.4 \%)\end{array}$ \\
\hline 52. & Hela-2468 & $-{ }^{-}$ & $-{ }^{-}$ & $-{ }^{-}$ & $-{ }^{-}$ & $4917 \pm 34$ & $5659-5600$ ВР (68.2\%) \\
\hline 53. & Hela-2475 & $-{ }^{-}$ & $-{ }^{-}$ & $-{ }^{-}$ & $-\ldots$ & $4854 \pm 34$ & $\begin{array}{l}5614-5583 \text { ВР }(52.2 \%) \\
5643-5629 \text { ВР }(11.8 \%) \\
5498-5492 \text { ВР }(4.3 \%)\end{array}$ \\
\hline
\end{tabular}


according to their absolute age. Depth maps were made for each spatially correlated paleosol surface. These maps, as well as separate radargrams, show that in particular points and zones the paleosols cross the groundwater table and descend below the present sea level (Fig. 4A). Thus, it is likely that the mentioned points and zones reflect the former position of lagoon coast of the Curonian Spit. Based on this assumption, a number of paleogeographic reconstructions of paleodune morphology for the different phases of dune development in the Dead (Grey) Dunes massif have been carried out using Halliburton Geographix's SeisVision program. The reconstructions have been created for smaller areas, depending on how widely the paleosols of a particular soil-forming generation were preserved. The LIDAR topography serves as comparative background for estimation of the dune evolution and the lagoon shoreline changes (Fig. 5).

\section{Results}

The investigations carried out by ground-penetrating radar enabled identification and mapping of paleosols and groundwater table (Fig. 4), and characterization of the inner structure of sand layers. In some instances, it was possible to differentiate coarse-grained marine sand from fine-grained lagoon sand enriched in organic matter (Fig. 2). All the mentioned geological peculiarities of sandy dunes near the earth surface were groundtruthed by control excavations and shallow handmade drillings. GPR images allowed to trace key sedimentary structures to depths of -20 meters.

All radiocarbon results ( 46 dates in total) range from approximately 5750 calendar years BP to the modern time (Table 1). It is possible to distinguish a number of soil-forming generations of different ages according to their age distribution (Fig. 6):
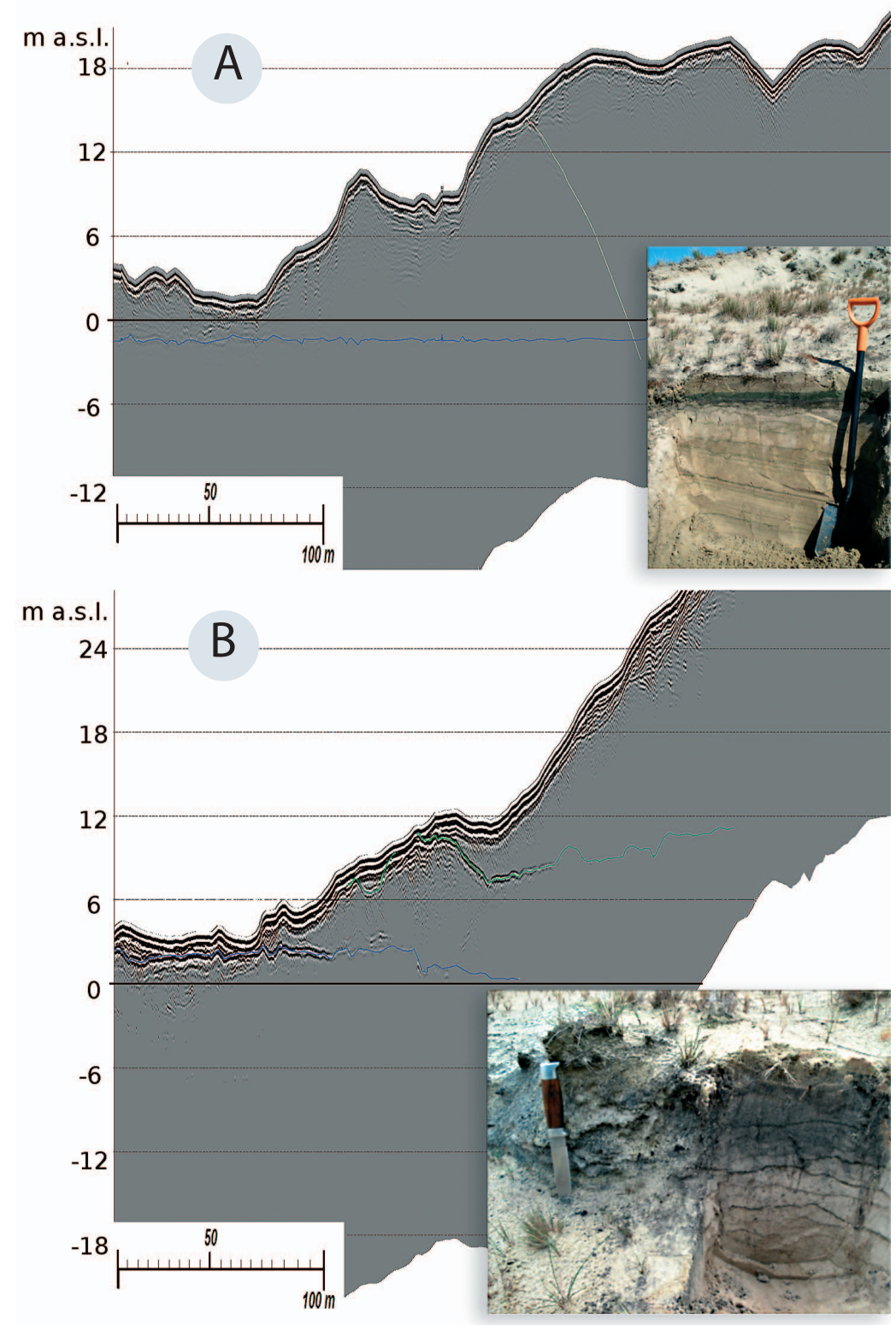

Fig. 4. Paleosols of different age in the GPR profiles and their context in excavations. Age of paleosols (Table 1): A (exposure No. 21), 523-426 calendar years BP; B (paleosol F), 3778-3678 calendar years BP. The groundwater table is indicated by the blue line. The buried paleosols are shown in violet $(A)$ and green $(B)$. The depth of excavations is $0.9(A)$ and $0.4(B)$ meters. 

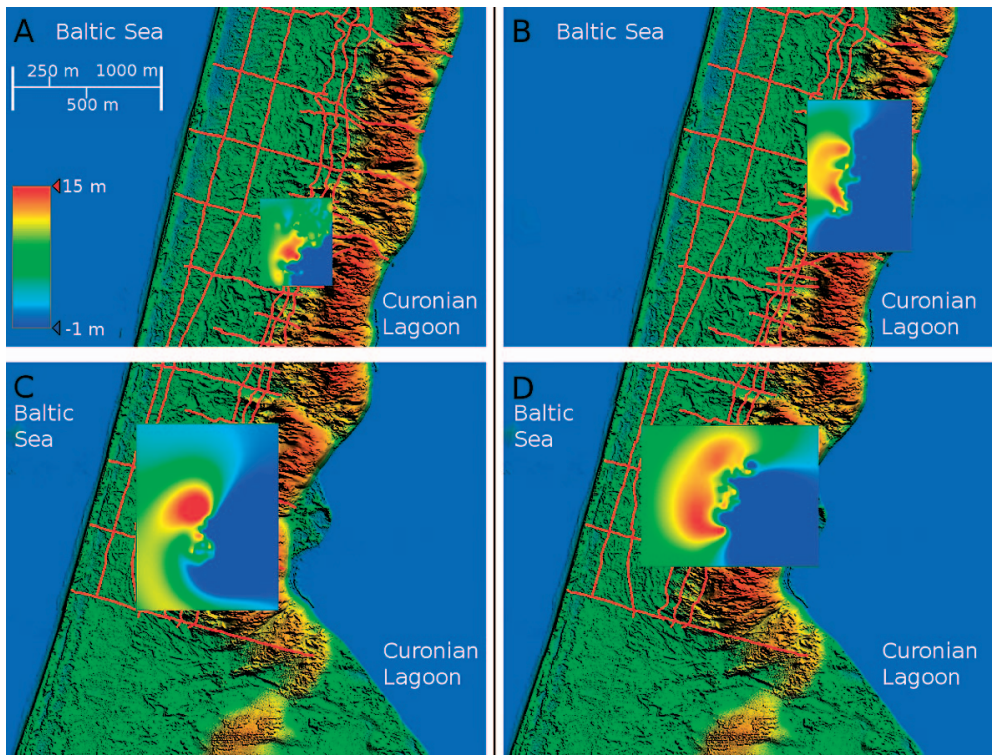

Fig. 5. Paleogeographic reconstructions of the Dead (Grey) Dunes massif for different points in time: A, C - about 3800-3500, B - about 430, and D about 1000 calendar years BP. The reconstructed fragments are shown as inserted rectangles; the remaining parts on the figures are filled in by LIDAR topography with georadar survey grid. Color height scale is valid for reconstructed paleorelief only. ancient lagoon coast were carried out only for limited areas in the southern part of the Dead Dunes (Fig. 5). According to these reconstructions, the coastline of the former Curonian Lagoon was approximately at the same position during all soil-forming periods, which was near the central (axial) part of the Curonian Spit, along the western slope of the present Great Dune Ridge. The highest altitudes of paleosols in the Curonian Spit were fixed at more than $40 \mathrm{~m}$ above present sea level (Michaliukaite, 1962), but according to our paleoreconstructions, based on the results of GPR survey, the height of paleodunes located along the paleocoasts in the southern part of Dead Dunes massif did not exceeded 15-20 meters.
1) 5800-4500, 2) 3900-3100, 3) 2600-2400, and

4) 1900 - modern (in calendar years BP). The time boundaries of these generations have been set very approximately, with an error of one hundred years, which is consistent with the timeframe of pedogenesis in temperate coastal settings. The last soil-forming generation contains a few paleosols of different age: it was confirmed by visual mapping during the fieldwork. Different layers of paleosols are also well visible in GPR radargrams. The absolute age of the aforementioned paleosols is very similar (the dates are overlapping), so the subdivision of the last soil-forming generation into shorter intervals is still problematic.

Geophysical surveys show that paleosols of all soil-forming generations in the Dead (Grey) Dune massif survived fragmentarily, mostly in the southern part of the massif, along the eastern slope of the Great Dune Ridge. Only a few small fragments of paleosols were detected in the central and northern parts of the investigated area. Thus, reliable paleogeographic reconstructions indicating morphology of paleodunes and former position of

\section{Discussion}

The paleogeographic reconstructions show that the ancient coast of the Curonian Lagoon during all the soil-forming periods was approximately close to the central part of the present Curonian Spit. Thus, it is possible to argue that for a long time span (from 6 kyr BP until the XVI century), the former dune massifs evenly covered the entire area of the Curonian Spit. They probably were not concentrated into one single sandy ridge, with a number of three-dimensional (e.g., parabolic) forms indicated by some paleosols. Thus, it could be concluded that the present Great Dune Ridge was formed starting only in the XVI century due to extremely high aeolian activity influenced by destructive human activities. A number of investigators considered the ancient forest fires as the main reason of re-activation of dune migration (Gudelis, 1998b; Gaigalas \& Pazdur, 2008). However, the resent investigations showed that wildfires probably stimulated the activity of aeolian 


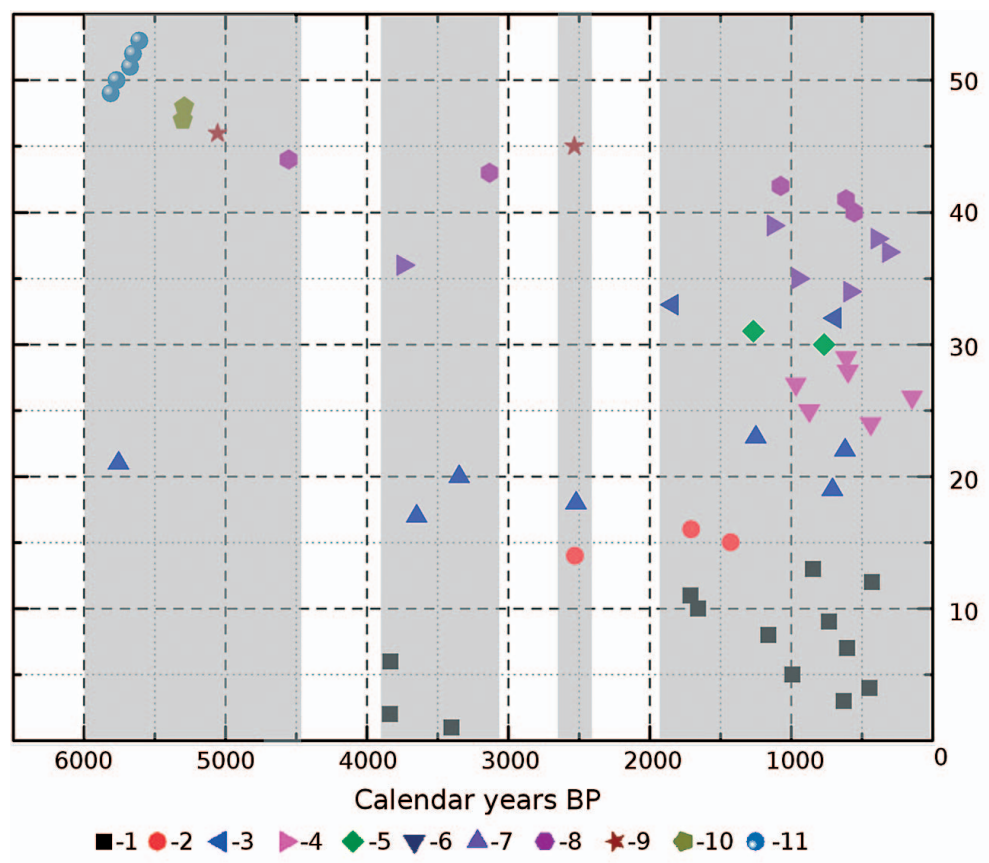

Fig. 6. Results of radiocarbon $\left({ }^{14} \mathrm{C}\right)$ dating of paleosols and archaeological artefacts in the Curonian Spit. Determinated the soil-forming generations are marked by grey shadow. The number in the Y-axis corresponds to the number of sample in Table 1.

Sources of dates: 1 - this study; 2 Bitinas et al., unpublished; 3 - Buynevich et al., unpublished; 4 - Gaigalas \& Pazdur, 2008; 5 - Buynevich et al., 2007; 6 - Moe et al., 2005; 7 - Gaigalas et al., 1991; 8 - Gerasimov \& Zavelski, 1980; Chichiagova \& Cherkinski, 1988; 9 - Gudelis \& Michaliukaite, 1976; 10 Rimantiene, 1999; 11 - Piliciauskas et al., 2011. processes only at very local scales. At that time there was not any significant displacement of the entire dune massifs. The distribution of the ancient aeolian massifs in the Curonian Spit possibly was similar as in the present Vistula Spit where the youngest dunes were concentrated in the seaward part of the Spit (Fedorowicz et al., 2012). In our opinion, a relatively young age of the Great Dune Ridge has been also confirmed by the analysis of cartographic materials. According to these data, the lagoon coast of the Skilvit dune massif in 1910 (the Russian part of the Curonian Spit) extended approximately along the present central part of the Great Dune Ridge (Povilanskas et al., 2009). From 1910-1912 the coastline of the lagoon have been removed up to 200-400 m eastward in other places of the Curonian Spit (Kazakevičius, 1989-1990). The aforementioned data maintain an extremely rapid advance of dune massifs during the past centuries.

The oldest radiocarbon dates of the paleosols in the Curonian Spit, i.e. about 5800 years BP, fit very well with the results of archaeological investigations, i.e. radiocarbon dating of artefacts. The oldest radiocarbon dates received by the archaeologists testified that about 5800 years BP the Curonian Spit was already populated
(Rimantiene, 1999; Piličiauskas et al., 2011), i.e. it happened immediately after the spit was overgrown and the first paleosol generation was established.

The spatial distribution of paleosols in the Dead Dunes massif and the results of radiocarbon dating indicate that the youngest soil-forming generation (from 1900 calendar years BP until the present; Fig. 6) consists of several soil-forming intervals of different age. The problem is that these "hidden" soil-forming generations are difficult to fit into the strict time limits because in many cases the dated paleosols nearest by age are "overlapping" each other. It depends on a few factors. The formation of well developed soil horizons in poorly vegetated sandy sediments of the Curonian Spit took place up to 1100-1600 years (Peyrat, 2007), so the different paleosols naturally "overlap" each other in age. On the other hand, the results of radiocarbon dating considerably depend on the dated material that has been subjectively selected by investigator. Thus, we can obtain different dates from the same paleosol layer. If a soil humus formed during the relatively long time interval (hundreds of years) was dated, an "averaged" date is obtained. In another case, if a charcoal fragment was dated, the obtained date represents relatively short period of late-stage soil 
formation (a few decades of tree life). Moreover, there is a possibility that single pieces of charcoal in the younger paleosols could be re-deposited by wind from older eroded paleosols. Detailed radiocarbon dating of different organic components in a single paleosol profile (i.e. methodological investigations) could solve this problem and help establish the optimal way for sampling and dating of paleosols. As a result, the correlation of paleosols and determination of soil-forming generations could be more reliable and precise. In addition, ongoing optical dating of aeolian sand horizons will help further constrain the radiocarbon chronology.

We suggest that during the Holocene, calm (soilforming) phases were regularly changed by episodes of aeolian activity (dune-forming), and these processes could be linked with climate variability, but such links are not always supported by the results of other investigations (Kotilainen, 2004; Raukas, 2011; and others). Moreover, in some areas, as in the Vistula Spit (Poland), phases of intense aeolian activity (Fedorowicz et al., 2012) fit well with the calm soil-forming periods in the Curonian Spit as determined during our investigations. Thus, the question of relationship between climate and dunes/ paleosols formation remains open to future investigations.

\section{Conclusions}

The paleosols representing past soil-forming generations in the Dead (Grey) Dune massif survived fragmentarily, mainly in the southern part of the massif, along the eastern slope of the Great Dune Ridge.

According to the results of radiocarbon $\left({ }^{14} \mathrm{C}\right)$ dating it is possible to distinguish four soil-forming generations (age in calendar years BP): 5800-4500, 3900-3100, 2600-2400, and 1900 - modern. The latest generation contains several paleosols of different age, but the more detailed subdivision of this pedogenic phase is not feasible at this time due to methodological problems of sediment dating.

The reliable paleogeographic reconstruction of paleodunes morphology and ancient coastlines of the Curonian Lagoon in the present Dead Dune massif is available only fragmentarily, in the very limited areas where the paleosols survived, i.e. in the southern part of the massif.

During all the soil-forming phases the ancient coastline of the Curonian Lagoon was, apart the minor shifts, approximately at the same position close to the central part of the present Curonian Spit, i.e. along the western slope of the Great Dune Ridge. The ancient dune massifs evenly covered the entire area of the Curonian Spit. The Great Dune Ridge has been formed only starting from the XVI century due to extremely high aeolian activity influenced by destructive human practices. The ancient forest fires stimulated only local re-activation of aeolian processes and did not trigger significant migration of the main dune massifs.

\section{Acknowledgments}

The research was funded by a grant (No. MIP-11131) from the Research Council of Lithuania. The project was supported by Lithuanian National Commission for UNESCO and by Direction of the Curonian Spit National Park. Cordial thanks to Ilya Buynevich (Temple University, USA) for fruitful consultations, language edits, and kind permission to use the results of paleosols dating. The authors wish to thank Rimas Petrošius, Žana Skuratovič and Nijole Skuodiene for assistance with the radiocarbon dating of paleosols, also express their gratitude to Dmitrij Gerok and Julius Aukštuolis for help in the field. Thanks to Hannes Tõnisson and an anonymous reviewer for critical remarks and positive evaluation of the manuscript.

\section{References}

Arslanov, K.A. 1985. Radiocarbon: Geochemistry and Geochronology. Leningrad. (in Russian)

Bučas, J. 2001. Curonian Spit National Park. Savastis, Vilnius. (in Lithuanian with English summary)

Bronk Ramsey, C., Dee, M., Lee, S., Nakagawa, T. \& Staff, R.A. 2010. Developments in the calibration and modelling of radiocarbon dates. Radiocarbon 52(3), 953-961.

Buynevich, I.V., Bitinas, A. \& Pupienis, D. 2007a. Lithological anomalies in a relict coastal dune: Geophysical and paleoenvironmental markers. Geophysical Research Letters 34, L09707.

Buynevich, I.V., Bitinas, A. \& Pupienis, D. 2007b. Reactivation of coastal dunes documented by subsurface imaging of the Great Dune Ridge, Lithuania. Journal of Coastal Research SI 50, 226-230. 
Buynevich, I.V., Jol, H.M. \& FitzGerald, D.M. 2009. Coastal Environments. In Jol, H.M. (ed.), GPR: Radar Theory and Applications. Elsevier, pp. 299-322.

Chichiagova, O.N. \& Cherkinski, A.E. 1988. Radiocarbon investigations in geographical survey. In: The materials of Radiometric Laboratory of Geographical Institute of Academy of Science of USSR, Part 1, Moscow, pp. 6-79. (in Russian)

Fedorowicz, S., Zieliński, P., Wysiecka, G. \& HoB ub, B. 2012. Phases of aeolian accumulation on the Vistula Spit (Southern Baltic Sea) in the light of TL dating and analysis of a digital elevation model. Geological Quarterly 56, 345352.

Gaigalas, A., Banys, J., Gulbinskas, S. \& Savukyniene, N. 1991. The radiocarbon age of the buried soils in the dunes of the Kuršiu Nerija spit. In: Gaigalas, A. (ed.) Geochronological and isotopic-geochemical investigations in the Quaternary geology and archaeology, Vilnius, pp. 813. (in Russian with English summary)

Gaigalas, A. \& Pazdur, A. 2008. Chronology of buried soils, forest fires and extreme migration of dunes on the Kuršiu nerija spit (Lithuanian coast). Landform Analysis 9, 187191.

Gerasimov, I.P. \& Zavelski, F.S. 1980. Radiocarbon investigations in the Radiometric Laboratory of Geographical Institute of Academy of Science of USSR. Bulletin of Quaternary Research Commission 50, 206 213. (in Russian)

Gudelis, V. 1998a. The Lithuanian offshore and coast of the Baltic Sea. Lithuanian Academy of Sciences Monograph, Vilnius. (in Lithuanian)

Gudelis, V. 1998b. A Catastrophic Dune Forest Fire on the Kuršiu Nerija Spit (Lithuanian Coast) and its Impact on the Coastal Population in the Late Neolithic Time. Pact $54,45-50$.

Gudelis, V. 1989-1990. Lithology of old parabolic dunes of the Curonian Spit and the coastal dynamics of the late Litorina Sea. The Geographical Yearbook 25-26, 13-17. (in Lithuanian)

Gudelis, V. \& Michaliukaite, E. 1976. The old parabolic dunes of the Curonian Spit. Geographia Lituanica, Vilnius, 5963. (In Russian)

Gudelis, V., Klimavičiene, V. \& Savukyniene, N. 1993. Old buried forest soils in the Kuršiu Nerija spit and their palynological pattern. In: Gudelis, V. (ed.) Baltic Sea coastal dynamics and paleogeography issues, Vol. 2, Academia, Vilnius, pp. 64-93. (in Lithuanian with English summary)

Gupta, S.H. \& Polach, H.A. 1985. Radiocarbon Practices at ANU, Handbook. Australian National University, Canberra.

Kazakevicius, S. 1989-1990. Dynamics of the Curonian Spit coastal development (cartographic analysis). Geographical
Yearbook 25-26, 46-56.

Kotilainen, M. 2004. Dune Stratigraphy as an Indicator of Holocene Climatic Change and Human Impact in Northern Lapland, Finland. Anales Academaiae Scientarium Fennicae, Geologica-Geographica, 166. Suomalainen Tiedeakatemia, Helsinki.

Kovaliukh, N.N. \& Skripkin, V.V. 1994. An universal technology for oxidation of carbon-containing materials for radiocarbon dating. In: Abstracts and Papers of Conference on Geochronology and Dendrochronology of Old Town's and Radiocarbon Dating of Archaeological Findings. Vilnius, Lithuania, 31 October-4 November, Vilnius University Press, pp. 37-42.

Michaliukaite, E. 1962. The old dunes and palaeosols of the Curonian Spit. The Geographical Yearbook 5, 377-390. (in Lithuanian)

Moe, D., Savukyniene, N. \& Stančikate, M. 2005. A new ${ }^{14} \mathrm{C}$ (AMS) date from former heathland soil horizons at Kuršiu Nerija, Lithuania. Baltica 18, 23-28.

Paul, K.H. 1944. Morphologie und Vegetation der Kurischen Nehrung. Nova Acta Leopoldina, 132 (96), Halle (Sante). (in German)

Peyrat, J. 2007. Development, properties and classification of dune soils in the Curonian Spit National Park, Russian part. Geologija 59, 59-64.

Piliciauskas, G., Lavento, M., Oinonen, M. \& Gri•as, G. 2011. New ${ }^{14} \mathrm{C}$ dates and early metal period ceramics in Lithuania. Radiocarbon 53, 629-643.

Povilanskas, R., Baghdasarian, H., Arakeylyan, S., Satkūnas, J. \& Taminskas, J. 2009. Secular morphodynamic trends of the Holocene dune ridge on the Curonian Spit. Journal of Coastal Research 25, 209-215.

Raukas, A. 2011. Evolution of aeolian landscapes in NorthEastern Estonia under environmental changes. Geographia Polonica 84, SI Part 1, 117-126.

Reimer, P.J., Baillie, M.G.L., Bard, E., Bayliss, A., Beck, J. W., Blackwell, P.G., Bronk Ramsey, C., Buck, C.E., Burr, G.S., Edwards, R.L., Friedrich, M., Grootes, P.M., Guilderson, T.P., Hajdas, I., Heaton, T.J., Hogg, A.G., Hughen, K.A., Kaiser, K.F., Kromer, B., McCormac, F.G., Manning, S. W., Reimer, R.W., Richards, D.A., Southon, J.R., Talamo, S., Turney, C.S.M., van der Plicht, J. \& Weyhenmeyer, C.E. 2009. IntCal09 and Marine09 radiocarbon age calibration curves, 0-50,000 years cal BP. Radiocarbon 51, 1111-1150.

Rimantiene, R. 1999. Curonian Spit at a glance of an archaeologist. Academy of Arts Press, Vilnius. (in Lithuanian)

Schumann, J. 1861. Die Wandernden Dunen auf Kurischen Nehrung. N. Preuss Prov., Blatt., 8. (in German)

Wichdorff, von H. 1919. Geologie der Kurischen Nehrung. Preuss. Abh. D. Preuss Geol. Landesanstalt, Neue Folge, H. 77. (in German) 